\title{
The quantitative genetics of sexual dimorphism: assessing the importance of sex-linkage
}

\author{
DJ Fairbairn and DA Roff \\ Department of Biology, University of California, Riverside, CA, USA
}

\begin{abstract}
Sexual dimorphism (SD) is a defining feature of gonochorous animals and dioecious plants, but the evolution of SD from an initially monomorphic genome presents a conundrum. Theory predicts that the evolution of SD will be facilitated if genes with sex-specific fitness effects occur on sex chromosomes. We review this theory and show that it generates three testable predictions. For organisms with an $X X / X Y$ chromosomal system of sex determination: (1) SD should be associated with X-linked effects; (2) X-linked effects should show strong directional dominance for sexually dimorphic traits favored in males but expressed in both sexes; and (3) SD should be associated with a reduction in the between-sex additive genetic covariance and correlation. A literature review reveals that empirical evaluations of the association between sex-linkage and SD have lagged behind theory. Tests for the presence of sex-linked effects have been
\end{abstract}

plagued by the need to make simplifying assumptions, such as the absence of dominance or maternal effects, that greatly weaken their discriminatory power. Further, most have used comparisons between species or populations, whereas the correct level of analysis is within populations. To overcome these problems, we derive a novel pedigree design that permits separate estimation of $\mathrm{X}$-linked, dominance and maternal effects. We suggest that the data from such a design would be most appropriately analyzed using the animal model. This novel protocol will allow quantitative evaluation of the above predictions, and hence should spur progress in understanding the role of sex-linkage in the evolution of SD.

Heredity (2006) 97, 319-328. doi:10.1038/sj.hdy.6800895; published online 30 August 2006

Keywords: sexual dimorphism; sex-linkage; diallel cross; animal model

\section{Introduction}

Sexual dimorphism (SD) is the defining feature of organisms in which male and female reproductive organs occur in different individuals (cf. gonochorous animals and dioecious plants). In most organisms, (SD) extends beyond the fundamental differentiation of organs of reproduction to include dimorphisms for body size, shape and color, as well as the presence of specific morphological structures in one sex only (e.g., sexlimited traits such as the antlers of male ungulates and the elaborate coloration of many male birds and teleost fishes; Darwin, 1871; Andersson, 1994; Short and Balaban, 1994). While 'dimorphism' in the strict sense refers to morphology, the term SD is also used for differences between the sexes in physiology, behavior and life history (Daly and Wilson, 1983; Short and Balaban, 1994; Mealey, 2000). In this broader sense, SD includes all aspects of the differentiation of males and females.

$\mathrm{SD}$ is presumed to reflect adaptive divergence in response to selection favoring different optimal character states in the two sexes (Hedrick and Temeles, 1989; Fairbairn, 1997; Badyaev and Hill, 2003; Blanckenhorn, 2005). This presents a conundrum for evolutionary

Correspondence: Dr DJ Fairbairn, Department of Biology, University of California, 900 University Avenue, Riverside, CA 92521, USA.

E-mail: daphne.fairbairn@ucr.edu

Received 4 May 2006; revised 13 July 2006; accepted 20 July 2006; published online 30 August 2006 biologists: how does SD evolve given the evolutionary constraints imposed initially by the shared genomes of the two sexual morphs (Lande, 1980, 1987; Reeve and Fairbairn, 1996, 1999, 2001; Fairbairn, 1997; Badyaev, 2002)? In some cases, the sexes can be so disparate as to be unrecognizable as the same species (Darwin, 1871) and yet these highly distinct phenotypes can arise from substantively identical genomes. Chromosomal sex determination is clearly not required, as extreme SD occurs in many animals that lack sex chromosomes (i.e., where sex is determined by environmental cues or in response to age or body size changes; Pieau et al., 1994; Shapiro, 1994; Berec et al., 2005). Nevertheless, evolutionary theory predicts that the evolution of SD will be facilitated if genes with sex-specific fitness effects occur on sex chromosomes or can be translocated there (e.g., Fisher, 1931; Charlesworth and Charlesworth, 1980; Rice, 1984; Charlesworth, 2002). Therefore, sex-linkage may be one solution to the conundrum presented above. This prediction is enticing not only in the context of the evolution of SD but also because it attributes additional functionality to the sex chromosomes beyond their primary role in sex determination.

Although the expectation of an association between SD and sex-linkage has a strong theoretical basis, formal tests of this theory have been limited by lack of appropriate experimental protocols. Here, we describe three predictions that can be derived from the underlying theory and critically review experimental protocols that have been used to test these predictions. We propose 
a new quantitative genetic protocol for separation of $\mathrm{X}$ linked additive variance from confounding maternal and dominance effects. Application of this protocol should permit formal, quantitative evaluation of the association between X-linkage and SD.

\section{Sex-linkage, sex-limited gene expression and the evolution of SD}

For organisms with chromosomal sex determination, genes on the sex chromosomes are responsible for initiating the cascade of developmental processes leading to SD (Lyon, 1994; Marshall Graves, 1994; Mealey, 2000; Bainbridge, 2003). Once the cascade is initiated, the responding genes need not be on the sex chromosomes and evidence from both humans and fruitflies (Drosophila melanogaster) indicates that indeed, most are distributed on autosomes (Saifi and Chandra, 1999; Parisi et al., 2003). However, several genetic models predict that the evolution of SD is facilitated by sex-linkage for genes with differential fitness effects in males and females (Fisher, 1931; Charlesworth and Charlesworth, 1980; Rice, 1984; Charlesworth et al., 1987; Reinhold, 1998, 1999; Hurst and Randerson, 1999; Gibson et al., 2002; Reeve and Pfennig, 2003; Parisi et al., 2003; Fitzpatrick, 2004). Such genes are expected to accumulate on the $X$ chromosome (or Z chromosome for organisms with ZZ/ ZW sex determination), and to show non-additive allelic interactions. The rationale for this prediction can be illustrated in a simplified fashion by a single-locus model with complete dominance in which females are the homogametic sex (females, XX; males, $\mathrm{XY}$ or $\mathrm{XO}$ ) (Figure 1). In such a system, selection for traits that benefit only males is expected to favor recessive alleles at $X$-linked loci because these are expressed more often in males, especially when rare. Dominant X-linked alleles are expressed more often in females, but here the differential is small and hence X-linkage is predicted

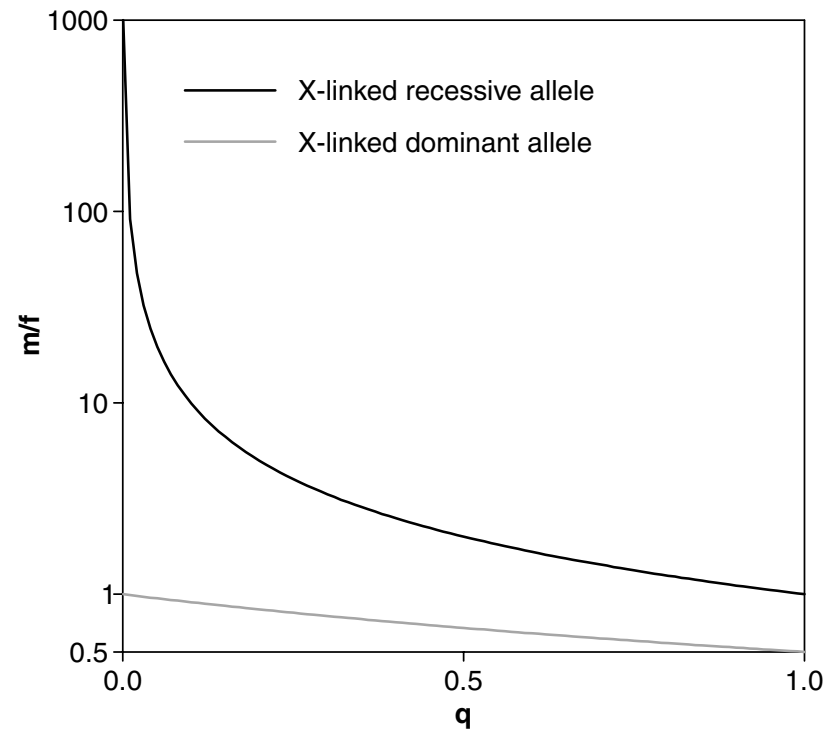

Figure 1 The expected ratio of the proportion of males $(\mathrm{m})$ and females ( $\mathrm{f}$ ) expressing $\mathrm{X}$-linked alleles as a function of the frequency of the recessive allele, $q$. For recessive alleles: $m / f=q / q^{2}=1 / q$. For dominant alleles: $\mathrm{m} / \mathrm{f}=p /\left(p^{2}+2 p q\right)=1 /(1+q)$. primarily for traits favored in males. Similar arguments lead to the prediction that X-linkage will be most strongly favored for traits whose expression is already limited to males (Reinhold, 1999; Andrés and Morrow, 2003).

While one might expect genes coding for traits favored in males to be found preferentially on the $\mathrm{Y}$ chromosome in XX/XY systems (e.g., Charlesworth et al., 1987; Chippindale and Rice, 2001; Reeve and Pfennig, 2003), Y chromosomes are often degenerate or even absent and $X$-linkage is therefore much more common than Y-linkage (Charlesworth and Charlesworth, 2000; Charlesworth, 2002).

Dosage compensation may influence these simple predictions in a number of ways (Charlesworth et al., 1987; Rogers et al., 2003; Carrel and Willard, 2005). The net effect of dosage compensation is to increase the additive genetic variance for X-linked traits in males relative to females, which should favor rapid evolutionary response of $X$-linked genes favored in males, as predicted (Charlesworth et al., 1987). However, Rogers et al (2003) have suggested that dosage compensation through up-regulation of X-linked genes in males, as in Drosophila melanogaster, enhances expression of deleterious X-linked mutations in males, and hence should favor translocation of genes with strong male-specific fitness effects to autosomes. Another complication arises if dosage compensation occurs through inactivation of genes on one $X$ chromosome in females (Rao and Arora, 1979; Carrel and Willard, 2005; Turner, 2006). If this renders females effectively hemizygous at X-linked loci, it will remove dominance effects, and hence remove the advantage of X-linked over autosomal alleles. Thus, although a considerable body of theory predicts an association between SD and X-linkage, our increasing appreciation of dosage compensation adds new complexity, rendering empirical evaluation of this prediction even more critical.

A second genetic mechanism expected to facilitate the evolution of SD is the evolution of sex-specific patterns of expression of autosomal genes (Lande, 1987; Rhen, 2000). This can occur through recruitment of alleles with sexlimited effects at existing loci, as well as through the evolution of new modifier genes and regulatory pathways (Lande, 1980, 1987; Rice, 1984; Rhen, 2000; Badyaev, 2002). Sex-specific gene expression and sex-linkage are likely to be functionally connected because sex-linked genes often exert their phenotypic effects by regulating the expression of autosomal genes (Charlesworth et al., 1987; Marshall Graves, 1994; Chippindale and Rice, 2001; Bainbridge, 2003). Hence, the two mechanisms are not mutually exclusive. Both are expected in organisms with chromosomal sex determination, although their relative importance may vary over the course of the evolutionary trajectory of SD (Lande, 1980, 1987; Rice, 1984).

\section{Three key predictions}

Three key predictions can be derived from the genetic hypotheses described above:

- Prediction 1: SD should be associated with X-linked effects. Specifically, the expected contribution of Xlinkage should be greatest for traits limited to males, intermediate for sexually dimorphic traits favored in 
males but expressed in both sexes, and least for sexually monomorphic traits. In the context of quantitative genetic variance, we would therefore expect the proportion of genetic variance that is X-linked to be positively correlated with the degree of SD. Note, however, that traits limited to females or favored only in females are excluded from these predictions.

- Prediction 2: X-linked effects should show strong directional dominance for sexually dimorphic traits favored in males but expressed in both sexes.

- Prediction 3: SD should be associated with reduction in the between-sex additive genetic covariance $\left(\mathrm{COV}_{\mathrm{Amf}}\right)$ and correlation $\left(r_{\mathrm{Amf}}\right)$, to the limit of $\mathrm{COV}_{\mathrm{Amf}} \rightarrow 0$ and $r_{\text {Amf }} \rightarrow 0$ for traits expressed in only one sex. Both sexlinkage and sex-specific expression of autosomal loci are expected to contribute to these effects, but the form and magnitude of the expected changes are difficult to predict. The genetic variance associated with X-linked loci is expected to differ between the sexes, even with most forms of dosage compensation (see above and Lynch and Walsh, 1998), and this can be expected to reduce the between-sex additive genetic covariance $\left(\mathrm{COV}_{\mathrm{Amf}}\right)$. Sex-limited autosomal gene expression is also expected to reduce $\mathrm{COV}_{\text {Amf }}$ (Reeve and Fairbairn, 2001). Most authors predict a similar trend in $r_{\mathrm{Amf}}$ (Fisher, 1958; Eisen and Legates, 1966; Lande, 1980, 1987; Arnold, 1985; Bonduriansky and Rowe, 2005), but this latter expectation is somewhat more tenuous because $r_{\text {Amf }}$ depends on the additive genetic variances in each sex in addition to the covariance and may not change in parallel with $\mathrm{COV}_{\mathrm{Amf}}$ (Reeve and Fairbairn, 2001).

These predictions are derived directly from the genetic models summarized above which assume large or infinite populations and continuous availability of new mutations. An alternative scenario, proposed by Meagher (1992), is that selection for SD could eventually deplete the genetic variance at loci with sex-linked or sex-limited effects, leaving most of the genetic variance segregating at autosomal loci expressed in both sexes. Such a scenario would lead to an increase in $\mathrm{COV}_{\mathrm{Amf}}$ and $r_{\text {Amf }}$ as SD evolves (Meagher, 1992), hence reversing prediction 3 . It would also eventually reduce the contribution of X-linked effects to the standing genetic variance for dimorphic traits, negating prediction 1 . The depletion of genetic variance inherent in Meagher's scenario is not a feature of most genetic models but may be realistic for natural systems if selection is strong and effective population sizes are low. X-linked variance may be particularly at risk, as $N_{e}$ is lower for $\mathrm{X}$ chromosomes than for autosomes (Gibson et al., 2002). The signature of such an effect would be falsification of prediction 1 combined with a positive rather than negative association between $\mathrm{SD}$, and $\mathrm{COV}_{\mathrm{Amf}}$ and $r_{\mathrm{Amf}}$.

\section{Previous empirical tests}

In this section, we review the conclusions reached from published experiments: we suspend discussion of the limitations of these conclusions resulting from underlying assumptions until the following section in which we review the experimental methods themselves. Much of the research on the genetic mechanisms facilitating the evolution of SD has focused on D. melanogaster. This species has an unusually large $X$ chromosome (Saifi and Chandra, 1999; Parisi et al., 2003; Fitzpatrick, 2004), a characteristic that one might expect would result in unusually high X-linked variance. Nevertheless, empirical evaluations of the prediction that SD should be associated with X-linkage in D. melanogaster have yielded conflicting results. Elegant line-cross analyses have revealed strong $X$-linkage for sexually antagonistic variation in adult fitness (Gibson et al., 2002) as well as for sexually selected behavioral and morphological traits (Reinhold, 1998). An extensive half-sib mating experiment (Cowley et al., 1986; Cowley and Atchley, 1988) uncovered significant additive X-linked variance for 22 of 28 linear morphological measures. These studies indicate strong effects of X-linked genes on the genetic variance of these traits. In contrast, genomic surveys and QTL studies have detected no bias towards X-linkage for genes influencing sexually selected behavioral traits in $D$. melanogaster (Gleason et al., 2002; Fitzpatrick, 2004). Further, a genome-wide survey of genes showing sex-biased expression revealed no tendency for those preferentially expressed in males to be on the $X$ chromosome (Parisi et al., 2003; Ranz et al., 2003). Reconciling these disparate conclusions is difficult: studies partitioning phenotypic and genetic variance for traits (Cowley et al., 1986; Cowley and Atchley, 1988; Reinhold, 1998; Gibson et al., 2002) find significant effects of X-linkage, while those documenting the physical locations of genes for SD traits (Gleason et al., 2002; Parisi et al., 2003; Ranz et al., 2003; Fitzpatrick, 2004) find no bias towards X-linkage. The solution may lie in the distinction between the physical location of a gene (on the sex chromosomes or autosomes) and the relative influence of that gene on trait expression. The results summarized above suggest that genes influencing sexually dimorphic traits are not found disproportionately on the $X$ chromosome, but that those on the $X$ chromosome exert a disproportionate influence on trait expression, particularly in males. Such an effect has been described for X-linked genes in humans (Saifi and Chandra, 1999) and may be at least partly explained by dosage compensation (Cowley et al., 1986; Charlesworth et al., 1987).

Evidence from other organisms also supports the hypothesis that SD is associated with the expression of $X$-linked genes, particularly for traits exaggerated in or limited to males. Reciprocal line crosses indicate significant sex-linked effects in many insect, bird and mammal species (Barbato, 1991; Reinhold, 1994, 1998, 2002; Wolfenbarger and Wilkinson, 2001). Although maternal effects may inflate these estimates in mammals, this is less likely in birds and insects (Wolfenbarger and Wilkinson, 2001; Reinhold, 2002). Genomic approaches have demonstrated that genes for traits associated with sex and reproduction (SRR traits) in male mammals tend to be X-linked (Saifi and Chandra, 1999; Wang et al., 2001), and in humans, these X-linked genes tend to have high penetrance and strong pleiotropic effects relative to autosomal genes (Saifi and Chandra, 1999).

Although these and other studies support the hypothesis that sex-linked genes play a role in the evolution of many sexually dimorphic traits, there is little doubt that most genes contributing to SD for polygenic traits are autosomal and show some degree of sex-specific expression. The major role played by this mechanism has been 
well established by studies of the developmental genetics and ontogeny of SD in species with chromosomal sex determination (Daly and Wilson, 1983; Short and Balaban, 1994; Mittwoch, 1996; Mealey, 2000; Badyaev, 2002; Skelly and John-Alder, 2002; Cox et al., 2005; Emlen et al., 2005; Ketterson et al., 2005). The discovery of similar developmental cascades and hormonal mechanisms in organisms with environmental sex determination (Pieau et al., 1994), including those that are sequentially hermaphroditic (Shapiro, 1994), provides unassailable evidence that sex-linkage is not required for the evolution of even extreme SD. The issue at hand is not whether sex-linked genes are required for the evolution of SD, but rather how much sex-linkage contributes to the standing genetic variance for these traits in organisms with sex chromosomes and whether this is proportional to the degree of SD (e.g., monomorphic vs dimorphic vs sexlimited).

The prediction that $\mathrm{COV}_{\mathrm{Amf}}$ should covary negatively with SD (prediction 3) has not been tested empirically, but the predicted negative relationship between $r_{\mathrm{Amf}}$ and SD has recently been confirmed for a series of morphological traits in the fly, Prochyliza xanthostoma (Bonduriansky and Rowe, 2005). Other studies from which one can extract both SD and $r_{\mathrm{Amf}}$ for a variety of traits are uncommon (e.g., Cowley et al., 1986; Cowley and Atchley, 1988; Meagher, 1992; Preziosi and Roff, 1998) and of these, only the estimates of $r_{\text {Amf }}$ for $D$. melanogaster (Cowley et al., 1986; Cowley and Atchley, 1988) include possible X-linked effects in males because these are excluded from standard sire estimates from half-sib designs. These data show no correlation between $\mathrm{SD}$ and $r_{\mathrm{Amf}}\left(R_{26}=-0.12, P>0.50\right)$ and the trend is opposite to that predicted. Additional evaluations of the relationship between $\mathrm{COV}_{\mathrm{Amf}}, r_{\mathrm{Amf}}$ and $\mathrm{SD}$ are clearly required before any general conclusions can be drawn.

\section{Experimental protocols: using crosses between populations to detect sex-linked effects}

The most frequently used method to detect the influence of sex-linkage is a reciprocal cross between two inbred or selected lines, populations or species (Hagger and Stranzinger, 1992; Reinhold, 1998, 2002; Wolfenbarger and Wilkinson, 2001; Table 1). Males from such crosses differ in their $X$ chromosome but not their autosomes, and differences between males from the two cross types are generally attributed to X-linked genes. However, this assumes that there are no maternal effects, no Y-linked effects in the case of XY males (Carson and Lande, 1984), and no effects of genomic imprinting (Spencer, 2002). The presence of maternal effects can be assessed from the difference between the reciprocal cross lines for the homogametic sex (females; italic font in Table 1). Both sex-linkage and maternal effects can then be estimated if it is assumed that the maternal effects are the same in both sexes. However, the addition of F1 reciprocal backcrosses and F2 reciprocal hybrids permits more robust separation of autosomal, sex-linked and maternal effects (Table 2). Estimates of these effects can then be made using either line-cross analysis (see below) or ANOVA with a priori contrasts (Table 3).
Table 1 Genomic composition of offspring from reciprocal crosses between two 'lines' (i.e., inbred lines, populations or species), designated as types $\mathrm{A}$ and $\mathrm{B}$

\begin{tabular}{|c|c|c|}
\hline \multirow[t]{2}{*}{ Male parent } & \multicolumn{2}{|c|}{ Female parent } \\
\hline & $q_{\mathrm{A}}$ & $q_{\mathrm{B}}$ \\
\hline$\sigma_{\mathrm{A}}$ & $\mathrm{X}_{\mathrm{A}} \mathrm{X}_{\mathrm{A}} \mathrm{AA}^{\mathrm{a}}$ & $X_{A} X_{B} A B$ \\
\hline & $\mathrm{X}_{\mathrm{A}} 0 \mathrm{AA}$ & $\mathrm{X}_{\mathrm{B}} \mathrm{OAB}$ \\
\hline$\sigma_{\mathrm{B}}$ & $X_{A} X_{B} A B$ & $\mathrm{X}_{\mathrm{B}} \mathrm{X}_{\mathrm{B}} \mathrm{BB}$ \\
\hline & $\mathrm{X}_{\mathrm{A}} 0 \mathrm{AB}$ & $\mathrm{X}_{\mathrm{B}} \mathrm{OBB}$ \\
\hline
\end{tabular}

Within each cell the top line shows the composition of the homogametic sex and the bottom line shows the composition of the heterogametic sex ( $\mathrm{X} 0$ in this case). Males shown in bold have sex chromosomes derived from different lines and hence demonstrate X-linkage. However, they also differ in the source of their maternal chromosomes. Differences between these males could therefore be due to both sex-linkage and maternal effects. Females shown in italics have the same genomic composition but differ in the source of their maternal chromosomes and hence differences between these females reveal maternal effects.

a Source of $X$ chromosomes (e.g., $X_{A}$ from a type $A$ parent) and autosomes (e.g., $\mathrm{AB}$ means one half derived from a type $\mathrm{A}$ parent and one half from a type $B$ parent).

Line-cross analysis is most frequently used to estimate the contribution of additive and non-additive (maternal, dominance and epistasis) effects using a joint scaling test to determine the most appropriate model (Mather and Jinks, 1982; Lynch and Walsh, 1998). Model fitting is hierarchical, beginning with a simple model, such as the additive model (i.e., the expected mean phenotype of the crosses is a simple linear function of the relative contributions of the parental genomes such that the mean population values of the different crosses fall on a line connecting the two parental means, top graph, Figure 2), and increasing its complexity only if the model fails the joint-scaling test. In the presence of sex-linked effects, the expected mean phenotypic values change (Figure 2), and the line-cross analysis can be expanded to estimate sex-linked, epistatic and maternal effects (e.g., Carbonell et al., 1985; Barbato, 1991; Barbato and Vasilatos-Younken, 1991; Polak and Starmer, 2001; Huttunen and Aspi, 2003).

Parameter estimation from line-cross analyses is made using least-squares regression (Lynch and Walsh, 1998). Models with many parameters may be difficult to distinguish: in particular, the effects of X-linkage can be somewhat confounded with maternal effects. To ameliorate this problem, we recommend testing for $\mathrm{X}$-linked and maternal effects using planned contrast analyses, as shown in Table 3 (e.g., see Wahlsten, 1979; de Belle and Sokolowski, 1987; Huttunen and Aspi, 2003). If X-linked or maternal effects are significant in these planned comparisons, the line-cross analysis can then be initiated with these parameters included in the regression model.

\section{Experimental protocols: quantifying sex-linked variance within populations}

While the reciprocal cross method can detect the presence of sex-linkage, it does not estimate the magnitude of sex-linked effects within populations (i.e., the genetic variances and covariances). As described previously, theoretical studies of the evolution of sex- 
Table 2 Schematic of crosses used to separate hereditary components in two populations (lines or species) in a species with XO males

\begin{tabular}{|c|c|c|c|c|c|c|c|}
\hline \multirow[t]{3}{*}{ Cross } & \multicolumn{2}{|c|}{ Parents $^{\mathrm{a}}$} & \multicolumn{4}{|c|}{ Offspring genotypic ratio(s) } & \multirow[t]{3}{*}{ Source of maternal effects } \\
\hline & \multirow[t]{2}{*}{ Dam } & \multirow[t]{2}{*}{ Sire } & \multicolumn{2}{|c|}{ Autosomes ${ }^{\mathrm{b}}$} & \multicolumn{2}{|c|}{ Sex chromosome } & \\
\hline & & & From dam & From sire & Female (XX) $A A: A B: B B$ & Male $(\mathrm{X} 0) A: B$ & \\
\hline \multicolumn{8}{|c|}{ Parental population $(A \times A$ and $B \times B)$} \\
\hline 1 & A & A & $1: 0$ & $1: 0$ & 1:0:0 & $1: 0$ & A \\
\hline 2 & B & B & $0: 1$ & $0: 1$ & 0:0:1 & $0: 1$ & $\mathrm{~B}$ \\
\hline \multicolumn{8}{|c|}{ Reciprocal $F 1$ hybrids $(A \times B$ and $B \times A)$} \\
\hline 3 & A & $\mathrm{B}$ & $1: 0$ & $0: 1$ & $0: 1: 0$ & $1: 0$ & A \\
\hline 4 & B & A & $0: 1$ & $1: 0$ & 0:1:0 & $0: 1$ & B \\
\hline \multicolumn{8}{|c|}{ Reciprocal backcrosses } \\
\hline 5 & A & $\mathrm{F} 1(\mathrm{AB}) 3$ & $1: 0$ & $1: 1$ & 1:0:0 & 1:0 & A \\
\hline 6 & A & F1 (BA) 4 & $1: 0$ & $1: 1$ & $0: 1: 0$ & 1:0 & A \\
\hline 7 & B & F1 (AB) 3 & $0: 1$ & $1: 1$ & 0:1:0 & $0: 1$ & B \\
\hline 8 & B & F1 (BA) 4 & $0: 1$ & $1: 1$ & $0: 0: 1$ & $0: 1$ & B \\
\hline 9 & F1 (AB) 3 & A & $1: 1$ & $1: 0$ & $1: 1: 0$ & $1: 1$ & $(\mathrm{AB})$ \\
\hline 10 & F1 (AB) 3 & B & $1: 1$ & $0: 1$ & $0: 1: 1$ & 1:1 & $(\mathrm{AB})$ \\
\hline 11 & F1 (BA) 4 & A & $1: 1$ & $1: 0$ & 1:1:0 & $1: 1$ & (BA) \\
\hline 12 & F1 (BA) 4 & B & $1: 1$ & $0: 1$ & $0: 1: 1$ & $1: 1$ & (BA) \\
\hline \multicolumn{8}{|c|}{ Reciprocal F2 hybrids $(F 1 \times F 1)^{\mathrm{c}}$} \\
\hline 13 & F1 (AB) 3 & F1 (AB) 3 & $1: 1$ & $1: 1$ & 1:1:0 & $1: 1$ & $(\mathrm{AB})$ \\
\hline 14 & F1 (AB) 3 & F1 (BA) 4 & $1: 1$ & $1: 1$ & $0: 1: 1$ & $1: 1$ & (AB) \\
\hline 15 & F1 (BA) 4 & F1 (BA) 4 & 1:1 & 1:1 & $0: 1: 1$ & 1:1 & (BA) \\
\hline 16 & F1 (BA) 4 & $\mathrm{~F} 1(\mathrm{AB}) 3$ & $1: 1$ & $1: 1$ & 1:1:0 & $1: 1$ & (BA) \\
\hline
\end{tabular}

${ }^{a}$ Number indicates the cross generating the particular genotype (e.g., 'F1(AB)3' means that this parent is an offspring from the cross in the row labeled ' 3 ').

${ }^{\mathrm{b}} \mathrm{A}: \mathrm{B}$ ratio.

${ }^{\mathrm{C}}$ Corrects error in Table 1 of de Belle and Sokolowski (1987).

Table 3 Some possible contrasts to distinguish effects due to autosomal (A), sex-linked (X) and maternal (M) effects in the crosses shown in Table 2

\begin{tabular}{|c|c|c|c|c|c|c|}
\hline \multirow[t]{2}{*}{ Contrast } & \multicolumn{3}{|c|}{ Females } & \multicolumn{3}{|c|}{ Males } \\
\hline & $A$ & $X$ & $M$ & $A$ & $X$ & $M$ \\
\hline 1 vs $5 ; 2$ vs $8 ; 9 \& 11$ vs $13 \& 16 ; 10 \& 12$ vs $14 \& 15$ & + & & & + & & \\
\hline 1 vs $3 ; 2$ vs 4 & & & & + & & \\
\hline 3 vs 4 & & & + & & + & + \\
\hline 5 vs $6 ; 7$ vs $8 ; 13$ vs $14 ; 15$ vs 16 & & + & & & & \\
\hline 9 vs $10 ; 11$ vs 12 & + & + & & + & & \\
\hline
\end{tabular}

' + ' indicates that the effect is detected by the contrast. It is assumed that males are XO or that Y-linked effects are negligible.

linkage and SD generate specific predictions with respect to X-linkage, directional dominance and $r_{\mathrm{Amf}}$ at the within-population level. Therefore, to adequately test these predictions, research should be directed at partitioning variances within populations. The three predictions can be addressed by apportioning the phenotypic variance into its genetic and environmental components and then further partitioning the genetic variance into additive, dominance, sex-linked and maternal effects.

One method of estimating X-linked genetic variance utilizes multiple reciprocal crosses between inbred lines, a design known as 'the diallel cross' (Griffing, 1956; Sheridan et al., 1968; Lynch and Walsh, 1998). The diallel method permits the partitioning of trait variance into additive genetic, dominance, reciprocal and environmental variances. However, in most applications of this design, sex-linked and maternal effects are confounded, both being contained within the reciprocal variance.
Further, the method assumes no sex-linked dominance, the key aspect of prediction 2. For example, to estimate sex-linked effects on bristle number in D. melanogaster, Sheridan et al (1968) used a complex diallel cross in which they necessarily assumed no sex-linked dominance effects and no maternal effects other than those produced as a result of sex-linkage. Zhu and Weir (1996) solved the latter problem by deriving a method to partition the phenotypic variance $\left(V_{\mathrm{P}}\right)$ into $V_{\mathrm{P}}=V_{\AA}^{\mathrm{a}}+$ $V_{\mathrm{D}}^{\mathrm{a}}+V_{\mathrm{A}}^{\mathrm{x}}+V_{\mathrm{M}}+V_{\mathrm{E}}$, where $V_{\mathrm{A}}^{\mathrm{a}}$ is the additive genetic variance from autosomal loci, $V_{\mathrm{D}}$ is the dominance variance from autosomal loci, $V_{A}^{\chi}$ is the additive genetic variance from sex-linked loci (assuming the male has only a single $\mathrm{X}$ chromosome), $V_{\mathrm{M}}$ is the maternal variance (excluding sex-linked effects) and $V_{\mathrm{E}}$ is the environmental variance. However, the diallel cross approach remains limited in its application because the production of inbred lines is not practical for most 

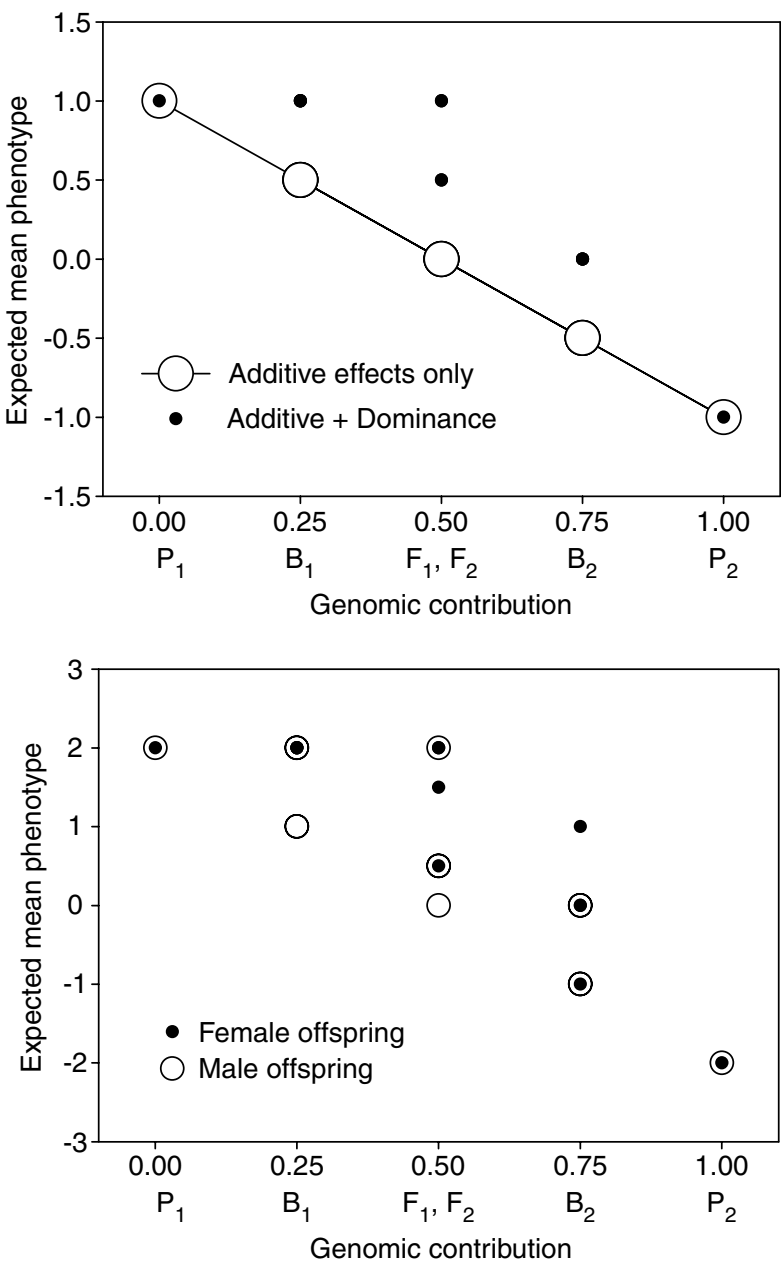

Figure 2 Schematic illustration of expected mean phenotypes from the line-cross analysis under the assumption of no X-linkage (top panel) and in the presence of X-linkage (bottom panel) with both additive and dominance effects. For simplicity epistasis and maternal effects are ignored. Using notation of Mather and Jinks (1982), $[d]=[h]=\left[d_{x}\right]=\left[h_{x}\right]=\left[d^{\prime}{ }_{x}\right]=1$, where $d$ refers to additive effects, $h$ to dominance effects and the subscript to X-linkage. Genomic contributions are indicated for parental $\left(P_{1}, P_{2}\right), F_{1}$ backcross $\left(B_{1}, B_{2}\right)$, and $F_{1}$ and $F_{2}$ hybrid generations.

animal species: Atchley and Zhu (1997) used the approach on inbred lines of mice but this is the only example we could locate. Further, the method of Zhu and Weir does not remove the problem of having to assume no sex-linked dominance effects.

Cowley et al (1986) and Cowley and Atchley (1988) used an alternative approach, a half-sib design, to estimate the sex-linked variance for a series of morphological traits in D. melanogaster. As their design utilized only one or two offspring per full-sib family, they had to assume, like Sheridan et al (1968), no dominance variance and no maternal effects. As noted above, the contribution of dominance variance is a key prediction of the theory and it should not be assumed to be absent. Maternal effects, if present, will inflate estimates of X-linkage. Hence, it is clearly preferable to estimate them directly, removing this component from the X-linked variance, rather than simply assuming its absence.

We suggest a new pedigree approach that is free from these assumptions. Our proposed design utilizes trait

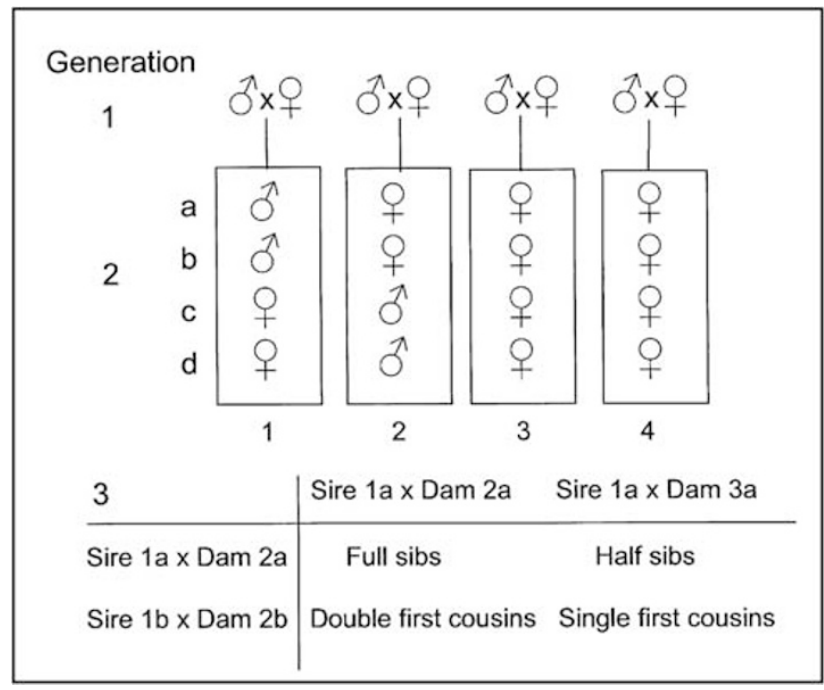

Figure 3 Schematic of one breeding set in the proposed threegeneration pedigree design. In generation 2 , each male mates with the three females on the same row. Relationships are illustrated for four sets of relatives in generation 3 (F1). All relationships are shown in Tables 4 and 5.

values from three consecutive generations to derive a full set of covariances among relatives. The basic breeding unit or 'set' is illustrated in Figure 3, and a given experiment would consist of many replicate sets initiated from the same population. (If possible, the number of sets should be determined from a power analysis based on a priori estimates of the (co)variance components.) Within each set, generation 1 consists of eight unrelated individuals that form four pairs of grandparents (GP). In generation 2 (parental generation, P), two full-sib male offspring from each of two GP pairs are used as sires which are mated to three unrelated dams, families 1 and 2 contributing two dams each and families 3 and 4 contributing four dams. This mating scheme produces sets of full sibs, half sibs, and single and double first cousins in generation 3 (F1).

To illustrate the procedure and why this novel mating design is necessary to obtain all variance components, we describe the framework for estimating heritabilities. To include sex-linked, maternal and dominance effects we have combined the maternal and sex-linkage models presented by Lynch and Walsh (1998). The covariance between relatives $x$ and $y$ is given by $\operatorname{Cov}_{x y}=$ $2 \Theta_{x y}^{\mathrm{a}} V_{\mathrm{A}}^{\mathrm{a}}+\Delta_{x y}^{\mathrm{a}} V_{\mathrm{D}}^{\mathrm{a}}+\phi_{x y}^{\mathrm{a}} V_{\mathrm{A}}^{\mathrm{x}}+c_{\mathrm{E}}^{\mathrm{m}} V_{\mathrm{E}}^{\mathrm{m}}+c_{\mathrm{A}}^{\mathrm{m}} V_{\mathrm{A}}^{\mathrm{m}}+c_{\mathrm{A}}^{\mathrm{ma}} \operatorname{Cov}_{\mathrm{A}}^{\mathrm{m}}$ for males, and $\operatorname{Cov}_{x y}=2 \Theta_{x y}^{\mathrm{a}} V_{\mathrm{A}}^{\mathrm{a}}+\Delta_{x \mathrm{y}}^{\mathrm{a}} V_{\mathrm{D}}^{\mathrm{a}}+2 \Theta_{x y}^{\mathrm{x}} V_{\mathrm{A}}^{\mathrm{x}}+\Delta_{x y}^{\mathrm{x}} V_{\mathrm{D}}^{\mathrm{x}}+$ $c_{\mathrm{E}}^{\mathrm{m}} V_{\mathrm{E}}^{\mathrm{m}}+c_{\mathrm{A}}^{\mathrm{m}} V_{\mathrm{A}}^{\mathrm{m}}+c_{\mathrm{A}}^{\mathrm{ma}} \operatorname{Cov}_{\mathrm{A}}^{\mathrm{m}}$ for females, where the superscript a denotes autosomal, $X$ the $X$-linked and $m$ the maternal components, and the subscript A denotes additive, $\mathrm{D}$ the dominance and $\mathrm{E}$ the environmental variance (e.g., $V_{\mathrm{A}}^{\mathrm{a}}$ is the additive genetic variance attributable to autosomal genes, $V_{\mathrm{D}}^{\mathrm{a}}$ is the autosomal dominance variance, $V_{\mathrm{A}}^{\times}$is the additive $\mathrm{X}$-linked variance, $V_{\mathrm{D}}^{\mathrm{x}}$ is the $\mathrm{X}$-linked dominance variance, $V_{\mathrm{E}}^{\mathrm{m}}$ is the maternal environmental variance, $V_{\mathrm{A}}^{\mathrm{m}}$ is the additive genetic maternal variance and $\operatorname{Cov}_{A}^{m}$ is the direct maternal additive genetic covariance). The two equations differ because of the possibility of non-additive gene action at X-linked loci in females. Utilizing the phenotypic values of sires and dams permits estimation of both sex-linked and maternal effects within each sex. Values 
Table 4 Coefficients for expressions describing the expected phenotypic covariance between male relatives in the proposed breeding design to estimate variances due to autosomal, sex-linked and sex-specific gene expression

\begin{tabular}{|c|c|c|c|c|c|c|c|}
\hline \multirow[t]{3}{*}{ Male-male relationships } & \multicolumn{7}{|c|}{ Coefficients } \\
\hline & \multicolumn{2}{|c|}{ Autosomal } & \multicolumn{2}{|c|}{ Sex-linked } & \multicolumn{3}{|c|}{ Maternal } \\
\hline & $V_{\mathrm{A}}^{\mathrm{a}}$ & $V_{\mathrm{D}}^{\mathrm{a}}$ & $V_{\mathrm{A}}^{\mathrm{X}}$ & $V_{\mathrm{D}}^{\mathrm{X}}$ & $V_{\mathrm{E}}^{\mathrm{m}}$ & $V_{\mathrm{A}}^{\mathrm{m}}$ & $\operatorname{Cov}_{\mathrm{A}}^{\mathrm{m}}$ \\
\hline 1. Father-Son & $1 / 2$ & 0 & 0 & & 0 & 0 & $1 / 4$ \\
\hline 2. Full brothers & $1 / 2$ & $1 / 4$ & $1 / 2$ & & 1 & 1 & 1 \\
\hline 3. Paternal half brothers & $1 / 4$ & 0 & 0 & & 0 & 0 & 0 \\
\hline 4. Single first cousins, dams full sisters & $1 / 8$ & 0 & $3 / 8$ & & 0 & $1 / 2$ & $1 / 2$ \\
\hline 5. Single first cousins, sires full brothers & $1 / 8$ & 0 & 0 & & 0 & 0 & 0 \\
\hline 6. Single first cousins, opposite sex full sibs & $1 / 8$ & 0 & 0 & & 0 & 0 & $1 / 4$ \\
\hline 7. Double first cousins, sires full brothers, dams full sisters & $1 / 4$ & $1 / 16$ & $3 / 8$ & & 0 & $1 / 2$ & $1 / 2$ \\
\hline 8. Double first cousins, opposite sex full sibs & $1 / 4$ & $1 / 16$ & 0 & & 0 & 0 & $1 / 2$ \\
\hline 9. Paternal uncle-nephew & $1 / 4$ & 0 & 0 & & 0 & 0 & $1 / 4$ \\
\hline 10. Maternal uncle-nephew & $1 / 4$ & 0 & 0 & & 0 & $1 / 2$ & $3 / 4$ \\
\hline 11. Paternal grandfather-grandson & $1 / 4$ & 0 & 0 & & 0 & 0 & $1 / 8$ \\
\hline 12. Maternal grandfather-grandson & $1 / 4$ & 0 & $1 / 2$ & & 0 & $1 / 4$ & $5 / 8$ \\
\hline
\end{tabular}

Modified (and corrected) from Tables 23.1, 24.1 of Lynch and Walsh (1998), Eisen (1967) and Grossman and Eisen (1989). Maternal dominance variance is assumed to be zero and environmental maternal effects are assumed to be transmitted only over one generation.

Table 5 Coefficients for expressions describing the expected phenotypic covariance between female relatives in the proposed breeding design to estimate variances due to autosomal, sex-linked and sex-specific gene expression

\begin{tabular}{|c|c|c|c|c|c|c|c|}
\hline \multirow{3}{*}{ Female-female relationships } & \multicolumn{7}{|c|}{ Coefficients } \\
\hline & \multicolumn{2}{|c|}{ Autosomal } & \multicolumn{2}{|c|}{ Sex-linked } & \multicolumn{3}{|c|}{ Maternal } \\
\hline & $V_{\mathrm{A}}^{\mathrm{a}}$ & $V_{\mathrm{D}}^{\mathrm{a}}$ & $V_{\mathrm{A}}^{\mathrm{X}}$ & $V_{\mathrm{A}}^{\mathrm{a}}$ & $V_{\mathrm{D}}^{\mathrm{a}}$ & $V_{\mathrm{A}}^{\mathrm{X}}$ & $V_{\mathrm{A}}^{\mathrm{a}}$ \\
\hline 13. Mother-daughter & $1 / 2$ & 0 & $1 / 2$ & 0 & 0 & $1 / 2$ & \\
\hline 14. Full sisters & $1 / 2$ & $1 / 4$ & $3 / 4$ & $1 / 2$ & 1 & 1 & 1 \\
\hline 15. Paternal half sisters & $1 / 4$ & 0 & $1 / 2$ & 0 & 0 & 0 & 0 \\
\hline 16. Single first cousins, dams full sisters & $1 / 8$ & 0 & $3 / 16$ & 0 & 0 & $1 / 2$ & $1 / 2$ \\
\hline 17. Single first cousins, sires full brothers & $1 / 8$ & 0 & $1 / 4$ & 0 & 0 & 0 & 0 \\
\hline 18. Single first cousins, opposite sex full sibs & $1 / 8$ & 0 & $1 / 8$ & 0 & 0 & 0 & $1 / 4$ \\
\hline 19. Double first cousins, sires full brothers, dams full sisters & $1 / 4$ & $1 / 16$ & $7 / 16$ & $3 / 16$ & 0 & $1 / 2$ & \\
\hline 20. Double first cousins, opposite sex full sibs & $1 / 4$ & $1 / 16$ & $1 / 4$ & $1 / 16$ & 0 & 0 & \\
\hline 21. Paternal aunt-niece & $1 / 4$ & 0 & $1 / 4$ & 0 & 0 & 0 & $1 / 4$ \\
\hline 22. Maternal aunt-niece & $1 / 4$ & 0 & $3 / 8$ & 0 & 0 & $1 / 2$ & $3 / 4$ \\
\hline 23. Paternal grandmother-granddaughter & $1 / 4$ & 0 & $1 / 2$ & 0 & 0 & 0 & $1 / 8$ \\
\hline 24. Maternal grandmother-grand daughter & $1 / 4$ & 0 & $1 / 4$ & 0 & 0 & $1 / 4$ & $5 / 8$ \\
\hline
\end{tabular}

Modified (and corrected) from Tables 23.1, 24.1 of Lynch and Walsh (1998), Eisen (1967) and Grossman and Eisen (1989). Maternal dominance variance is assumed to be zero and environmental maternal effects are assumed to be transmitted only over one generation.

of the coefficients for various relationships are shown in Tables 4 and 5 . For males, the relationships indicated by numbers $1,2,3,7,8$ and 12 form the minimum set from which all variance components can be estimated. For females, the minimum set of relationships includes those indicated by numbers 14, 15, 16, 19, 20, 22, 23 and 24 . Additional relationships contribute to the precision of the estimation by further limiting the range of possible values.

\section{Parameter estimation using the animal model}

In a design such as the above pedigree analysis, involving several generations, there will often be more than one way to estimate a particular variance component. While one could make separate estimates, a method that combines all the information into a single procedure is clearly more desirable. The analytical method known as 'the animal model' accomplishes this (Lynch and Walsh, 1998; Kruuk, 2004). The animal model approach uses each individual as a datum in contrast to more traditional models, such as the half-sib design, which estimate sire values from the values of their progeny. To illustrate the animal model, consider a simple case in which only additive effects are present: in this case the phenotypic value of all individuals is given by $\mathbf{y}=\beta+\boldsymbol{\mu}+\mathbf{e}$, where $\mathbf{y}$ is the vector of trait values for all individuals, $\beta$ is the population mean, $\boldsymbol{\mu}$ is a vector of additive genetic effects and $\mathbf{e}$ is a vector of residual errors. The advantages of the animal model are that it can accommodate very complex pedigrees and it estimates all variance and covariance components using the entire data set. Further, variance and covariance components are generally estimated using restricted maximum likelihood, which permits the testing of different models using the log-likelihood ratio test. An additional bootstrap approach to estimate parameter values and standard errors is advisable to test the adequacy of the maximum likelihood model: a close correspondence with the restricted maximum likelihood estimates can be taken as evidence that the latter approach is adequate. 
Previous applications of the animal model to both nondomestic and domestic animals have assumed maternal effects but no sex-linkage. In the simplest extension of the animal model, maternal effects are fitted by making dam identity a random effect (e.g., Kruuk et al., 2000; Milner et al., 2000). Parameter estimation in domestic breeds has frequently included the maternal additive genetic variance, direct maternal additive genetic covariance and maternal environmental variance (e.g., see Clément et al., 2001) and this model was fitted to the feral population of Soay sheep (Wilson et al., 2005). Additionally, non-additive variances have been estimated for domestic breeds (e.g., Rodriguez-Almeida et al., 1995). The theory for the construction of the relevant matrices for the inclusion of sex-linked effects has been worked out by Grossman and Fernando (1989) and Fernando and Grossman (1990). However, a citation search revealed no citations to this work and, to the best of our knowledge, the animal model has not yet been used to estimate sex-linked variance (personal communication: Loeske Kruuk, University of Edinburgh; William Hill, University of Edinburgh; Denis Reale, Université du Québec à Montréal). Given the proposed wide occurrence of sexlinked effects in SD traits and the bias introduced by use of an incomplete genetic model (Clément et al., 2001), we recommend inclusion of sex-linked effects whenever the traits examined are sexually dimorphic.

The general approach of the animal model is to fit a model with all components and compare this to reduced models using the likelihood ratio test (e.g., Reale et al., 1999; Wilson et al., 2005). For example, although maternal effects are likely to be negligible for most morphological traits (Mousseau and Roff, 1987; Reinhold, 2002), this expectation is insufficient justification for an a priori assumption of no effect. Use of the animal model approach requires no such assumption. We can test for maternal effects by comparing the full model to a model in which maternal effects are assumed to be absent. The same approach can be used to test for sex-linked effects: the full model would be compared to one without sexlinked effects included.

\section{Using within-population variance components to test the three key predictions}

Once the required variance components and covariances have been estimated, as proposed above, the three predictions presented earlier can be recast in a statistical framework and tested. For purposes of illustration and simplicity, we assume an $X X / X Y$ or $X X / X O$ chromosomal system, no Y-linked effects, and no epistasis. To test for a positive association between $X$-linked variance and SD (prediction 1), we are interested in the proportion of the genetic variance attributable to $X$-linked variance. Males cannot show $X$-linked dominance variance, so the relevant parameter is $V_{\mathrm{A}}^{\mathrm{x}} /\left(V_{\mathrm{A}}^{\mathrm{x}}+V_{\mathrm{A}}^{\mathrm{a}}+V_{\mathrm{D}}^{\mathrm{a}}\right)$ for males, while it is $\left(V_{\mathrm{A}}^{\mathrm{x}}+V_{\mathrm{D}}^{\mathrm{x}}\right) /\left(V_{\mathrm{A}}^{\mathrm{x}}+V_{\mathrm{D}}^{\mathrm{X}}+V_{\AA}^{\mathrm{a}}+V_{\mathrm{D}}^{\mathrm{a}}\right)$ for females. The second hypothesis, that $X$-linked effects should show directional dominance, can be tested by direct estimation of $V_{\mathrm{D}}^{\mathrm{x}}$, using a likelihood ratio test to compare a model in which $V_{\mathrm{D}}^{\mathrm{x}}=0$ for the trait under test with a model in which $V_{\mathrm{D}}^{\mathrm{X}}$ is free to vary. Since the prediction applies only to traits favored in males but still expressed in both sexes (i.e., not sex-limited), the expectation is that $V_{\mathrm{D}}^{\mathrm{X}}$ will be larger for these traits. The final prediction, that the additive genetic covariance and correlation between sexes should decline as SD increases, simply requires standard estimation of $\mathrm{COV}_{\mathrm{Amf}}$ and $r_{\mathrm{Amf}}$ (Lynch and Walsh, 1998).

Each of these predictions is relative rather than absolute: we predict an association between the genetic parameter of interest and the degree of SD rather than merely presence/absence of the variance component of interest. Statistical evaluation of the predictions therefore requires estimates for a series of traits differing in SD. Such a series could consist of traits differing in SD within a single species, an approach analogous to the comparative studies of various size traits in D. melanogaster (Cowley et al., 1986; Cowley and Atchley, 1988) and Aquarius remigis (Preziosi and Roff, 1998). However, if sufficient resources are available, extension of this singlespecies approach to comparisons of selected lines or closely related species would provide an even stronger test of the hypothesis, particularly if SD varies greatly both among traits and among comparison groups.

\section{Concluding remarks}

In spite of a robust and well-established body of theory, we still know relatively little about the evolutionary genetics of sexually dimorphic traits. We still do not know how much sex-linkage contributes to the standing genetic variance for these traits or whether this is proportional to the degree of SD. We do not know how or if the between-sex genetic covariances and correlations are related to SD, although predictions of both positive and negative associations exist in the literature. Perhaps most elusive of all, we have not yet begun to determine whether sex-linked effects are characterized by directional dominance. Progress on all of these questions has been hampered by lack of robust empirical methodologies for isolating and quantifying sex-linked effects. The hierarchical, pedigree breeding design that we have proposed is one step in addressing this problem. This method is an improvement over previous designs not only because it partitions the additive and dominance components of the sex-linked variance but also because these components are clearly separated from potentially confounding maternal and autosomal dominance effects. The resulting pedigree data can be analyzed using the animal model approach, which provides powerful and flexible hypothesis testing.

The proposed pedigree design should be suitable for many organisms, since it requires only a minimum of three generations of standardized rearing rather than the availability of inbred lines or interfertile species. Ideally, one should estimate sex-linked effects for a suite of traits ranging from sexually monomorphic, through sexually dimorphic to sex-limited within a given population. If resources permit, this approach can be extended to comparisons of the estimated parameters among selected lines or closely related species. This comparative approach would be particularly worthwhile if the range of dimorphisms within populations is small, but among species (or lines) is large. Scaling will be an issue in any of these analyses, as variances are scale-dependent. All measurements should therefore be on the same scale or transformed to a common scale. Strong genetic correlations among traits within a suite may bias statistical 
conclusions and limit the power of the comparisons to detect differences between traits. Avoidance of replicate traits within strongly integrated developmental units and a priori, statistical removal of integrative variables such as body size may help to ameliorate this problem.

The evolution of SD has long presented a conundrum for evolutionary biologists. In spite of an established body of predictive theory in this area, little empirical progress has been made. We hope that by summarizing the state of the field and providing a feasible experimental protocol for testing the existing hypotheses, we will inspire new research in this area.

\section{Acknowledgements}

We thank Loeske Kruuk, William Hill and Denis Reale for helpful advice, and Jeff Reeve for many insightful discussions during the early formulation of these ideas. We are also grateful for the constructive comments of two reviewers.

\section{References}

Andersson M (1994). Sexual Selection. Princeton University Press: Princeton, NJ.

Andrés JA, Morrow EH (2003). The origin of interlocus sexual conflict: is sex-linkage important? J Evol Biol 16: 219-223.

Arnold SJ (1985). Quantitative genetic models of sexual selection. Experientia 41: 1296-1309.

Atchley WR, Zhu J (1997). Developmental quantitative genetics, conditional epigenetic variablitiy and growth in mice. Genetics 147: 765-776.

Badyaev AV (2002). Growing apart: an ontogenetic perspective on the evolution of sexual size dimorphism. TREE 17: 369-378.

Badyaev AV, Hill GE (2003). Avian sexual dichromarism in relation to phylogeny and ecology. Ann Rev Ecol Evol System 34: $27-49$.

Bainbridge D (2003). The $X$ in Sex. How the $X$ Chromosome Controls Our Lives. Harvard University Press: Cambridge, MA.

Barbato GF (1991). Genetic architecture of growth curve parameters in chickens. Theor Appl Genet 83: 24-32.

Barbato GF, Vasilatos-Younken R (1991). Sex-linked and maternal effects on growth in chickens. Poult Sci 70: 709-718.

Berec L, Schembri PJ, Boukal DS (2005). Sex determination in Bonellia viridis (Echiura: Bonelliidae): population dynamics and evolution. Oikos 108: 473-484.

Blanckenhorn WU (2005). Behavioral causes and consequences of sexual size dimorphism. Ethology 111: 977-1016.

Bonduriansky R, Rowe L (2005). Intralocus sexual conflict and the genetic architecture of sexually dimorphic traits in Prochyliza xanthostoma (Diptera: Piophilidae). Evolution 59: 1965-1975.

Carbonell EA, Frey JJ, Bell AE (1985). Estimation of maternal, sex-linked and additive $\times$ additive epistatic gene effects for body size of Tribolium. Theor Appl Genet 70: 133-137.

Carrel L, Willard HF (2005). X-inactivation profile reveals extensive variability in $\mathrm{X}$-linked gene expression in females. Nature 434: 400-404.

Carson HL, Lande R (1984). Inheritance of a secondary sexual character in Drosophila silvestris. Proc Natl Acad Sci 81: 69046907.

Charlesworth B, Charlesworth D (2000). The degeneration of the Y chromosome. Philos Trans Roy Soc B 355: 1563-1572.

Charlesworth B, Coyne JA, Barton N (1987). The relative rates of evolution of sex chromosomes and autosomes. Am Nat 130 113-146.
Charlesworth D (2002). Plant sex determination and sex chromosomes. Heredity 88: 94-101.

Charlesworth D, Charlesworth B (1980). Sex differences in fitness and selection for centric fusions between sex chromosomes and autosomes. Gen Res 35: 205-214.

Chippindale AK, Rice WR (2001). Y chromosome is a strong determinant of male fitness in Drosophila melanogaster. Proc Natl Acad Sci 98: 5677-5682.

Clément V, Bibé B, Verrier É, Elsen J-M, Manfredi E, Bouix J et al. (2001). Simulation analysis to test the influence of model adequacy and data structure on the estimation of genetic parameters for traits with direct and maternal effects. Genet Sel Evol 33: 369-395.

Cowley DE, Atchley WR (1988). Quantitative genetics of Drosophila melanogaster. II. Heritabilities and genetic correlations between sexes for head and thorax traits. Genetics 119 421-433.

Cowley DE, Atchley WR, Rutledge JJ (1986). Quantitative genetics of Drosophila melanogaster. I. Sexual dimorphism in genetic parameters for wing traits. Genetics 114: 549-566.

Cox RM, Skelly SL, Leo A, John-Alder HB (2005). Testosterone regulates sexually dimorphic coloration in the eastern fence lizard, Sceloporus undulatus. Copeia 2005: 597-608.

Daly M, Wilson M (1983). Sexual development and differentiation. In: Daly M, Wilson M (eds) Sex, Evolution, and Behavior. Wadsworth: Belmont, CA. pp 243-277.

Darwin C (1871). The Descent of Man and Selection in Relation to Sex (reprinted in the Portable Darwin. DM Porter, PW Graham (eds). Penguin: London, 1993).

de Belle JS, Sokolowski MB (1987). Heredity of rover/sitter: alternative foraging strategies of Drosopila melanogaster larvae. Heredity 59: 73-83.

Eisen EJ (1967). Mating designs for estimating direct and maternal genetic variances and direct-maternal genetic covariances. Can J Genet Cytol 9: 13-22.

Eisen EJ, Legates JE (1966). Genotype-sex interaction and the genetic correlation between the sexes for body weight in $M u s$ musculus. Genetics 54: 611-623.

Emlen DJ, Hunt J, Simmons LW (2005). Evolution of sexual dimorphism and male dimorphism in the expression of beetle horns: phylogenetic evidence for modularity, evolutionary lability, and constraint. Am Nat 166: S42-S68.

Fairbairn DJ (1997). Allometry for sexual size dimorphism: pattern and process in the coevolution of body size in males and females. Ann Rev Ecol Syst 28: 659-687.

Fernando RL, Grossman M (1990). Genetic evaluation with autosomal and X-chromosomal inheritance. Theor Appl Genet 80: 75-80.

Fisher RA (1931). The evolution of dominance. Biol Rev 6: 345-368.

Fisher RA (1958). The Genetical Theory of Natural Selection 2nd edn. Dover Publishers: NY.

Fitzpatrick MJ (2004). Pleiotropy and the genomic location of sexually-selected genes. Am Nat 163: 800-808.

Gibson JR, Chippindale AK, Rice WR (2002). The X chromosome is a hot spot for sexually antagonistic fitness variation. Proc Roy Soc (Lond) B 269: 499-505.

Gleason JM, Nuzhdin SV, Ritchie MV (2002). Quantitative trait loci affecting a courtship signal in Drosophila melanogaster. Heredity 89: 1-6.

Griffing B (1956). A generalized treatment of the use of diallel crosses in quantitative inheritance. Heredity 10: 31-50.

Grossman M, Eisen EJ (1989). Inbreeding, coancestry, and covariance between relatives for X-chromosomal loci. Heredity 80: 137-142.

Grossman M, Fernando RL (1989). Covariance between relatives for X-chromosomal loci in a population in disequilibrium. Theor Appl Genet 77: 311-319.

Hagger C, Stranzinger G (1992). A method to detect large influences of specific sex chromosomes on quantitative traits. Theor Appl Genet 83: 925-929. 
Hedrick AV, Temeles EJ (1989). The evolution of sexual dimorphism in animals: hypotheses and tests. TREE 4: 136-138.

Hurst LD, Randerson JP (1999). An eXceptional chromosome. Trends Genet 15: 383-385.

Huttunen S, Aspi J (2003). Complex inheritance of male courtship song characters in Drosophila virilis. Behav Genet 33: $17-24$.

Ketterson ED, Nolan Jr V, Sandell M (2005). Testosterone in females: mediator of adaptive traits, constraint on sexual dimorphism, or both? Am Nat 166: S85-S98.

Kruuk LEB (2004). Estimating genetic parameters in natural populations using the 'animal model'. Philos Trans Roy Soc (London) B 359: 873-890.

Kruuk LEB, Clutton-Brock TH, Slate J, Pemberton JM, Botherstone S, Guiness FE (2000). Heritability of fitness in a wild mammal population. Proc Nat Acad Sci 97: 698-703.

Lande R (1980). Sexual dimorphism, sexual selection and adaptation in polygenic characters. Evolution 34: 292-305.

Lande R (1987). Genetic correlations between the sexes in the evolution of sexual dimorphism and mating preferences. In: Bradbury JW, Andersson MB (eds) Sexual Selection: Testing the Alternatives. John Wiley and Sons Ltd: New York. pp 83-94.

Lynch M, Walsh B (1998). Genetics and Analysis of Quantitative Traits. Sinauer Assocs: Sunderland, MA.

Lyon MF (1994). Evolution of mammalian sex chromosomes. In: Short AV, Balaban E (eds) The Differences Between the Sexes. Cambridge University Press: Cambridge, UK. pp 381-396.

Marshall Graves JA (1994). Mammalian sex-determining genes In: Short RV, Balaban E (eds) The Differences Between the Sexes. Cambridge University Press: Cambridge, UK. pp 397-418.

Mather K, Jinks JL (1982). Biometrical Genetics. Chapman \& Hall: London.

Meagher T (1992). The quantitative genetics of sexual dimorphism in Silene latifolia (Caryophyllaceae). I. Genetic variation. Evolution 46: 445-457.

Mealey L (2000). Sex Differences: Developmental and Evolutionary Strategies. Academic Press: San Diego, CA.

Milner JM, Pemberton JM, Brotherstone S, Albon SD (2000) Estimating variance components and heritabilities in the wild: a case study using the 'animal model' approach. J Evol Biol 13: 804-813.

Mittwoch U (1996). Sex-determining mechanisms in animals. TREE 11: 63-67.

Mousseau TA, Roff DA (1987). Natural selection and the heritability of fitness components. Heredity 59: 181-197.

Parisi M, Nuttall R, Naiman D, Bouffard G, Malley J, Andrews J et al. (2003). Paucity of genes on the Drosophila X chromosome showing male-biased expression. Science 299: 697-700.

Pieau C, Girondot M, Desvages G, Dorizzi, M Richard-Mercier N, Zaborski P (1994). Environmental control of gonadal differentiation. In: Short RV, Balaban E (eds) The Differences Between the Sexes. Cambridge University Press: Cambridge, UK. pp 433-448.

Polak M, Starmer WT (2001). The quantitative genetics of fluctuating asymmetry. Evolution 55: 498-511.

Preziosi RF, Roff DA (1998). Evidence of genetic isolation between sexually monomorphic and sexually dimorphic traits in the water strider Aquarius remigis. Heredity 81: 92-99.

Ranz JM, Castillo-Davis CI, Meiklejohn CD, Hartl DL (2003). Sex-dependent gene expression and the evolution of the Drosophila transcriptome. Science 300: 1742-1745.

Rao SRV, Arora P (1979). Insect sex chromosomes III. Differential susceptibility of homologous $X$ chromosomes of Gryllotalpa fossor to ${ }^{3} \mathrm{HUrd}$-induced aberrations. Chromosoma 74: 241-252.

Reale D, Festa-Bianchet M, Jorgenson JT (1999). Heritability of body mass varies with age and season in wild bighorn sheep Heredity 83: 526-532.
Reeve HD, Pfennig DW (2003). Genetic biases for showy males: are some genetic systems especially conducive to sexual selection? Proc Natl Acad Sci 100: 1089-1094.

Reeve JP, Fairbairn DJ (1996). Sexual size dimorphism as a correlated response to selection on body size: an empirical test of the quantitative genetic model. Evolution 50: 19271938.

Reeve JP, Fairbairn DJ (1999). Change in sexual size dimorphism as a correlated response to selection on fecundity. Heredity 83: 697-706.

Reeve JP, Fairbairn DJ (2001). Predicting the evolution of sexual size dimorphism. J Evol Biol 14: 244-254.

Reinhold K (1994). Inheritance of body and testis size in the bushcricket Poecilimon veluchianus Ramme (Orthoptera; Tettigoniidae) examined by means of subspecies hybrids. Biol $J$ Linn Soc 52: 305-316.

Reinhold K (1998). Sex linkage among genes controlling sexually selected traits. Behav Ecol Sociobiol 44: 1-7.

Reinhold K (1999). Evolutionary genetics of sex-limited traits under fluctuating selection. J Evol Biol 12: 897-902.

Reinhold K (2002). Maternal effects and the evolution of behavioral and morphological characters: a literature review indicates the importance of extended maternal care. J Hered 93: 400-405.

Rhen T (2000). Sex-limited mutations and the evolution of sexual dimorphism. Evolution 54: 37-43.

Rice WR (1984). Sex chromosomes and the evolution of sexual dimorphism. Evolution 38: 735-742.

Rodriguez-Almeida FA, Van Vleck LD, Willham RL, Northcutt SL (1995). Estimation of non-additive genetic variances in three synthetic lines of beef cattle using an animal model. I Anim Sci 73: 1002-1011.

Rogers DW, Carr M, Pomiankowski A (2003). Male genes: X-pelled or X-cluded? BioEssays 25: 739-741.

Saifi GM, Chandra HS (1999). An apparent excess of sex- and reproduction-related genes on the human $\mathrm{X}$ chromosome. Proc Roy Soc (London) B 266: 203-209.

Shapiro DY (1994). Sex change in fishes - how and why?. In: Short RV, Balaban E (eds) The Differences Between the Sexes. Cambridge University Press: Cambridge, UK. pp 105-130.

Sheridan AK, Frankham R, Jones LP, Rathie KA, Barker JSF (1968). Partitioning of variance and estimation of genetic parameters for various bristle number characters of Drosphila melanogaster. Theor Appl Genet 38: 179-187.

Short RV, Balaban E (1994). The Differences between the Sexes. Cambridge University Press: Cambridge, UK.

Skelly SL, John-Alder HB (2002). Effects of testosterone on growth and behavior of the eastern fence lizard (Sceloporus undulatus). Int Comp Biol 42: 1314

Spencer HG (2002). The correlation between relatives on the supposition of genomic imprinting. Genetics 161: 411-417.

Turner JMA (2006). X-inactivation: close encounters of the $X$ kind. Curr Biol 16: R259-R261.

Wahlsten D (1979). A critique of the concepts of heritability and heredity in behavioral genetics. In: Royce JR, Mos L (eds) Theoretical Advances in Behavioral Genetics. Sijthoff and Nordoff: Germantown, MD. pp 426-481.

Wang PJ, McCarrey JR, Yang F, Page DC (2001). An abundance of X-linked genes expressed in spermatogonia. Nat Genet 27: $422-426$.

Wilson AJ, Coltman DW, Pemberton JM, Overall AD, Byrne KA, Kruuk LE (2005). Maternal genetic effects set the potential for evolution in a free-living vertebrate population. J Evol Biol 18: $405-414$

Wolfenbarger LL, Wilkinson GS (2001). Sex-linked expression of a sexually selected trait in the stalk-eyed fly, Cyrtodiopsis dalmanni. Evolution 55: 103-110.

Zhu J, Weir BS (1996). Diallel analysis for sex-linked and maternal effects. Theor Appl Genet 92: 1-9. 